\title{
Crystal structure of oxalate decarboxylase from Photorhabdus luminescens, a symbiotic bacterium associated with entomopathogenic nematodes
}

\author{
Sreeja Chellappan ${ }^{1,2}$, S. Mathivanan ${ }^{1}$, R. Thippeswamy ${ }^{3}$, M. Nagesh ${ }^{3}$, \\ H. S. Savithri ${ }^{4}$ and M. R. N. Murthy ${ }^{1,5,6, *}$ \\ ${ }^{1}$ Molecular Biophysics Unit and ${ }^{4}$ Department of Biochemistry, Indian Institute of Science, Bengaluru 560012 , India \\ ${ }^{2}$ Department of Molecular Biology, Kannur University, Kannur 671 314, India \\ ${ }^{3}$ Indian Council of Agricultural Research, Project Directorate of Biological Control, Bengaluru 560 024, India \\ ${ }^{5}$ Institute of Bioinformatics and Applied Biotechnology, Bengaluru 560 100, India \\ ${ }^{6}$ Indian Institute of Science Education and Research, Thiruvananthapuram 695 551, India
}

Photorhabdus luminescens is a Gram-negative, symbiotic bacterium associated with entomopathogenic nematodes of the genus Heterorhabditis. Several genes from this organism related to insecticidal properties are being examined for their potential in pest management. Oxalate decarboxylase (OXDC), an enzyme secreted by bacteria and fungi and putatively associated with insecticidal pathways catalyses the manganese dependent decarboxylation of oxalate to formate and $\mathrm{CO}_{2}$. In this study, we report the $\mathrm{X}$-ray crystal structure of $O X D C$ isolated and purified from Photorhabdus luminescens (PlOXDC, MW $43 \mathrm{kDa})$ determined at $1.97 \AA$ resolution. PlOXDC protomer has a bicupin structure. Each cupin domain consists of two antiparallel $\beta$ sheets organized as a sandwich with a $\mathrm{Mn}^{2+}$ ion bound at the active site. PlOXDC exists as a mixture of monomeric and trimeric forms in solution but assumes a trimeric form in the crystal structure.

Keywords: Bicupin, crystal structure, oxalate decarboxylase, Photorhabdus luminescens.

PHOTORHABDUS LUMINESCENS is a Gram-negative entomopathogenic member of family Enterobacteriaceae ${ }^{1}$, existing in a symbiotic relationship, specifically with entomopathogenic nematodes belonging to family Heterorhabditiae (Phylum: Nematoda) ${ }^{2}$ Entomophagous nematodes with their symbiotically associated bacteria have been used as biological control agents to reduce the dependence on synthetic pesticides. The nematode juveniles harbour the bacterium in their gut, and enter the larva of host insect and release the bacteria into the insect haemocoel (body cavity). The bacteria rapidly multiply, secreting an array of toxins, antibiotics, proteolytic and lipolytic enzymes that cause larval mortality, prevention of putrefaction and bioconversion of the host larva which

*For correspondence. (e-mail: mrn@iisc.ac.in) promotes nematode development and multiplication. Duchaud et $\mathrm{al}^{3}{ }^{3}$ have determined the complete sequence of $P$. luminescens. Analysis of the genomic sequence revealed that it possesses a number of genes encoding for potential virulence factors.

Oxalate decarboxylase (OXDC), proposed to have insecticidal effect and secreted by bacteria, catalyses the manganese-dependent decarboxylation of oxalate to formate and $\mathrm{CO}_{2}$. OXDC was first reported from fungi Flammulina (Collybia) velutipes and Coriolus hersutus ${ }^{4}$. Later it was found in several other organisms ${ }^{5-12}$. OXDC structures have been determined from Bacillus subtilis and Thermotoga maritima ${ }^{13,14}$. These structures belong to the cupin superfamily of proteins which also includes some seed storage proteins ${ }^{15-17}$. Weerth et al. ${ }^{18}$ reported the structure of protein of unknown function but structurally similar to cupin fold proteins from Photorhabdus luminescens subsp. laumondii TTO1.

In this article, we report the X-ray crystal structure of Photorhabdus luminescens (isolated from entomopathogenic nematode, Heterorhabditis indica) oxalate decarboxylase (PlOXDC). The structural similarities and differences between $P l O X D C$ and other OXDCs are also discussed.

\section{Materials and method}

\section{Strain identification}

Bacterial culture was isolated and established from infective juveniles (IJs) of $H$. indica isolate NBAII HPL104 and then cultured on MacConkey's agar and NBTA plates for identification ${ }^{19}$. Pure monoxenic culture was grown in LB medium and genomic DNA was extracted using a DNA isolation kit (Sigma Bacterial Genomic DNA isolation kit). Purity of the culture was checked with 16s rRNA gene sequences. The $16 \mathrm{~S}$ rDNA sequence of 
P. luminescens strain NBAII HPL104 was deposited in the NCBI GenBank database under accession number JF923810.1. Full-length oxalate decarboxylase gene was amplified using the gDNA, sequenced and deposited in GenBank with accession number JN116589.1, along with its protein counterpart with accession number AEK20853.1.

\section{Expression and purification of oxalate decarboxylase}

PlOXDC was cloned and expressed in Escherichia coli BL21-DE3 cells. PlOXDC gene was amplified from $P$. luminescens genomic DNA using appropriate primers (forward primer: 5'-TGG AAC AAT TCC CGA TAT CTG AAG G-3' and reverse primer: 5'-TGC CCT CCG AT CAA TAA TGG TAA T-3'). The PCR product was inserted between BamHI and XhoI sites of the pGX4T2 vector, resulting in a GST tag covalently linked to the $\mathrm{N}$-terminus of $\mathrm{PlOXDC}$. The pre-inoculum was grown overnight at $37^{\circ} \mathrm{C}$ in the presence of $100 \mu \mathrm{g} / \mathrm{ml}$ of ampicillin (preculture). Next, $500 \mathrm{ml}$ of LB medium containing $100 \mu \mathrm{g} / \mathrm{ml}$ ampicillin was inoculated with $1 \%$ of the preculture and incubated at $30^{\circ} \mathrm{C}$ until the $\mathrm{OD}_{600}$ reached approximately 0.6 . This was followed by induction using $0.5 \mathrm{mM}$ IPTG. The culture was incubated overnight at $15^{\circ} \mathrm{C}$. The cells were harvested by centrifugation at $5000 \mathrm{~g}$ and resuspended in $30 \mathrm{ml}$ of cold lysis buffer (PBS with $2 \mathrm{mM}$ PMSF, $1 \mathrm{mM}$ DTT, $1 \%$ Triton X-100 and $2 \mathrm{mM}$ EDTA, pH 7.4), and sonicated for $15 \mathrm{~min}$. Cell debris was removed by centrifugation and the clear supernatant was used for further purification. The protein was purified by GST-tag affinity chromatography using GSTresin beads. The column consisting of bound OXDC was washed with $100 \mathrm{ml}$ of PBS containing $2 \mathrm{mM}$ EDTA to remove unbound proteins. It was further washed with buffer containing $20 \mathrm{mM}$ Tris (pH 8), $150 \mathrm{mM} \mathrm{NaCl}$ and $2 \mathrm{mM} \mathrm{MnCl}_{2}$ to remove other non-specifically bound proteins. Then $10 \mathrm{ml}$ of the same buffer and four units of thrombin $\left(\right.$ Novagen ${ }^{\circledR}$ ) were added to the column and incubated for $16 \mathrm{~h}$ at room temperature $\left(28^{\circ} \pm 2^{\circ} \mathrm{C}\right)$. The cleaved enzyme was eluted using $20 \mathrm{mM}$ Tris ( $\mathrm{pH}$ 8) containing $150 \mathrm{mM} \mathrm{NaCl}$ and $2 \mathrm{mM} \mathrm{MnCl}_{2}$. The protein obtained was further purified by gel filtration chromatography using a S200 Sephadex FPLC column. The purified protein was concentrated to $5 \mathrm{mg} / \mathrm{ml}$ using a $30 \mathrm{kDa}$ cut-off centricon. The expression and purity of the sample was checked by SDS-PAGE and further confirmed by $\mathrm{N}$-terminal sequencing. Accurate molecular mass was obtained by MALDI-MS.

\section{Enzyme activity measurements}

OXDC activity was estimated using the method of Bergmeyer and Grassl ${ }^{20}$, with minor modifications. The assay was carried out in micro-titre plates. The reaction mixture consisted of $10 \mathrm{mM}$ potassium acetate and $13 \mathrm{mM}$ potassium oxalate $(\mathrm{pH} 4)$. The reaction was started by the addition of various concentrations of PlOXDC ranging from 0.4 to $18.7 \mu \mathrm{g}$. The reaction mixture was incubated at $37^{\circ} \mathrm{C}$ for $5 \mathrm{~min}$ and then the reaction was stopped by the addition of $200 \mu \mathrm{l}$ of $150 \mathrm{mM}$ potassium phosphate buffer, $\mathrm{pH}$ 9.5. The amount of formate produced by the PlOXDC-catalysed reaction was estimated by coupling it to a formate dehydrogenase-catalysed reaction by adding $20 \mu \mathrm{l}$ of $57 \mathrm{mM} \mathrm{NAD}$ and $40 \mu \mathrm{l}$ of $40 \mathrm{U} / \mathrm{ml}$ formate dehydrogenase (final volume of assay mixture was $300 \mu \mathrm{l})$. Increase in absorbance at $340 \mathrm{~nm}$ due to NADH produced was monitored at $37^{\circ} \mathrm{C}$ until a constant value was reached (at about $30 \mathrm{~min}$ ), which ensured that all the formate formed in the first reaction was utilized by the second enzyme. One unit of OXDC activity was defined as micromoles of NADH formed per minute per milligram of enzyme under the assay conditions. Significant increase in absorbance by the product $\mathrm{NADH}$ was observed only when $1 \mu \mathrm{g}$ or more of PlOXDC was used in the assay, suggesting that the enzyme activity is low.

\section{Crystallization, data collection and structure determination}

Initial crystallization trials of native OXDC were performed by micro-batch method using Hampton crystal screens 1 and 2. Crystallization drops contained $2.5 \mu \mathrm{l}$ enzyme $(5 \mathrm{mg} / \mathrm{ml})$ and $2.5 \mu \mathrm{l}$ of the condition. Initial hits were obtained in condition nos 20,30 and 61 of the Hampton crystal screen. Only crystals which appeared in condition no. 30 (0.2 $\mathrm{M}$ ammonium sulphate and 30\% PEG 8000) were of quality sufficient for X-ray structure determination. A grid of conditions (ammonium sulphate 0.1-0.2 $\mathrm{M}$ and PEG $800025-35 \%$ ) around the original hit was used in further crystallization trials. The same set of conditions was used to co-crystallize the enzyme with substrates and analogues. For co-crystallization, enzyme at a concentration of $5 \mathrm{mg} / \mathrm{ml}$ was incubated with $50 \mathrm{mM}$ ligands. Although several liganded structures were determined, in most of them ethylene glycol (EDO), which was used as the cryo-protectant, was found at the active site. These structures were not included in further analysis.

Crystals of OXDC were soaked briefly in the mother liquor containing $20 \%$ EDO or glycerol as the cryoprotectant and flash-frozen in a stream of nitrogen gas at $100 \mathrm{~K}$. The crystals diffracted X-rays to $2.7 \AA$ resolution when examined using $\mathrm{X}$-rays from a rotating anode generator (home source, wavelength of $1.54 \AA$ ). Diffraction data on these crystals were collected to a resolution of $1.97 \AA$ using BM14 beam line (wavelength $0.9 \AA$ ) at the European Synchrotron Radiation Facility (ESRF), Grenoble, France. The data collected using home-source 
X-rays were indexed, integrated and scaled using iMOSFLM and SCALA programs of the CCP4 suite ${ }^{21}$. The scaled data were used for structure determination by molecular replacement employing the Phaser program ${ }^{22}$. Initial phasing model was a polyalanine chain of $B$. subtilis OXDC (BsOXDC; PDB code 1J58) with which PlOXDC shares a sequence identity of $49 \%$. This structure was later used as the starting model for molecular replacement structure determination in Phaser with the synchrotron data (1.97 $\AA$ resolution) processed using the HKL2000 package ${ }^{23}$. Since these structures correspond to the wild-type protein briefly exposed to the cryoprotectant EDO, only the higher resolution structure corresponding to the synchrotron data was retained. Modelbuilding and refinement were carried out using COOT $^{24}$ and REFMAC $5^{25}$ respectively. Table 1 provides the statistics of data collection and structure refinement.

\section{Results}

\section{Protein purification and characterization}

PlOXDC was purified using GST-tag affinity chromatography. GST tag was later removed by thrombin digestion. Based on the cloned sequence, the estimated mole-

Table 1. Statistics of processing for data collected on $P l O X D C$ crystals

\begin{tabular}{ll}
\hline Parameters & PlOXDC-EDO \\
Wavelength $(\AA)$ & 0.9 \\
Space group & $P 2{ }_{1}{ }_{1} 2_{1}$ \\
Unit cell parameters & \\
$\quad a, b, c(\AA)$ & $66.01,105.61,150.53$ \\
$\quad \alpha, \beta, \gamma\left({ }^{\circ}\right)$ & 909090 \\
Resolution $(\AA)^{\mathrm{a}}$ & $39.95-1.97(2.07-1.97)$ \\
$R_{\text {sym }}(\%)^{\mathrm{b}}$ & $7.8(43.5)$ \\
Mean $[\langle I\rangle / \sigma(\langle I\rangle)]$ & $14.7(3.9)$ \\
Completeness $(\%)$ & $90(83.2)$ \\
Multiplicity & $6.6(6.1)$ \\
Total no. of reflections & $451,498(54,857)$ \\
Unique reflections & $67,963(9039)$ \\
Matthews coefficient $\left(\AA^{3} / \mathrm{Da}\right)$ & 2.03 \\
Protomers in the ASU & 3 \\
rSolvent content $(\%)$ & 39.56 \\
Refinement statistics & \\
$R_{\text {work }}(\%)^{\mathrm{c}}$ & 20.2 \\
$R_{\text {free }}(\%)^{\mathrm{c}}$ & 27.0 \\
Protein/water/ligand & $2927 / 45 / 25$ \\
Bond length $(\AA)$ & 0.015 \\
Bond angle $\left({ }^{\circ}\right)$ & 1.867 \\
Favoured region & 95.0 \\
Allowed region & 3.9 \\
Outliers & 1.1 \\
\hline
\end{tabular}

${ }^{\mathrm{a}}$ Values in parentheses correspond to the highest resolution shell.

${ }^{\mathrm{b}} R_{\text {sym }}=\sum_{h} \sum_{\mathrm{j}}\left|I_{h j}-\left\langle I_{h}\right\rangle\right| / \sum_{j} \sum_{h} I_{h j}$, where $I_{h j}$ is the $j$ th measurement of the intensity of reflection $h$ and $\left\langle I_{h}\right\rangle$ is its average intensity.

${ }^{\mathrm{c}} R_{\text {work }}=\sum\left(\left|F_{\text {obs }}\right|-\left|F_{\text {calc }}\right|\right) / \sum\left|F_{\mathrm{o}}\right| . R_{\text {free }}$ was calculated similarly using $5 \%$ of the reflections that were excluded from the refinement. cular weight (MW) of the protein was $43 \mathrm{kDa}$. The PlOXDC protomeric MW estimated from SDS-PAGE was $\sim 40 \mathrm{kDa}$ (Figure $1 \mathrm{a}$ ), while the major peak observed in gel filtration corresponded to a species of MW $\sim 120 \mathrm{kDa}$, suggesting that $P l O X D C$ is probably a trimer in solution (Figure $1 b$ ). MW estimated by MALDI-TOF was $40,222 \mathrm{Da}$, suggesting that the enzyme may have lost a $3 \mathrm{kDa}$ peptide due to a non-specific proteolysis by thrombin or some other contaminating protease during purification (Figure $1 c$ ).

Gel filtration performed immediately after purification indicated that the major fraction of the protein was in trimeric form. However, a minor peak that corresponded to a monomeric species was also observed (Figure $1 \mathrm{~b}$ ). Therefore, the enzyme appears to exist as a mixture of monomeric and trimeric forms in solution, although the dominant species is the trimeric form. Upon prolonged storage (up to four weeks), monomeric species increased and the two forms were of similar abundance (data not shown).

\section{Enzyme activity}

Optimum temperature and $\mathrm{pH}$ for the activity were found to be $45^{\circ} \mathrm{C}$ and 3 respectively. The specific activity measurements suggested that the enzyme was not as active as OXDCs from other sources.

The $K_{\mathrm{m}}$ and $V_{\max }$ values were calculated to be $0.5 \mathrm{mM}$ and $7.8 \mu \mathrm{mol} \mathrm{min} \mathrm{mg}^{-1}$ respectively, by fitting Michaelis and Menten equation to the kinetic data. The $K_{\mathrm{m}}$ of $P l O X D C$ was lower when compared to that of $B s \mathrm{OXDC}$ (16.4 mM). $V_{\max }$ of PlOXDC was also three-fold lower

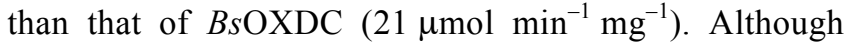
the enzyme was truncated as suggested by SDS-PAGE and MALDI experiments, it was active suggesting that the deleted segment is dispensable for the function of the enzyme. However, it is plausible that the reduced activity of $P l O X D C$ is a result of polypeptide truncation.

\section{Protomeric structure of PlOXDC}

All crystals used in the present study belong to the orthorhombic space group $P 2{ }_{1} 2_{1} 2_{1}$. The crystallographic asymmetric unit contained three protomers that are related by a nearly exact non-crystallographic three-fold axis of symmetry (Figure $2 a$ ) corresponding to a calculated Matthews coefficient of $2.03 \AA 3 \mathrm{Da}^{-1}$ (Table 1). No density was found for the $47 \mathrm{~N}$-terminal residues in any of the electron density maps. From the SDS-PAGE and MALDI results, it may be concluded that the enzyme is truncated by 26 residues. N-terminal sequencing showed that the protein is cleaved between A26 and A27 (data not shown). However, density corresponding to the additional 21 residues $(27-47)$ at the N-terminus was not observed in the electron density maps. 

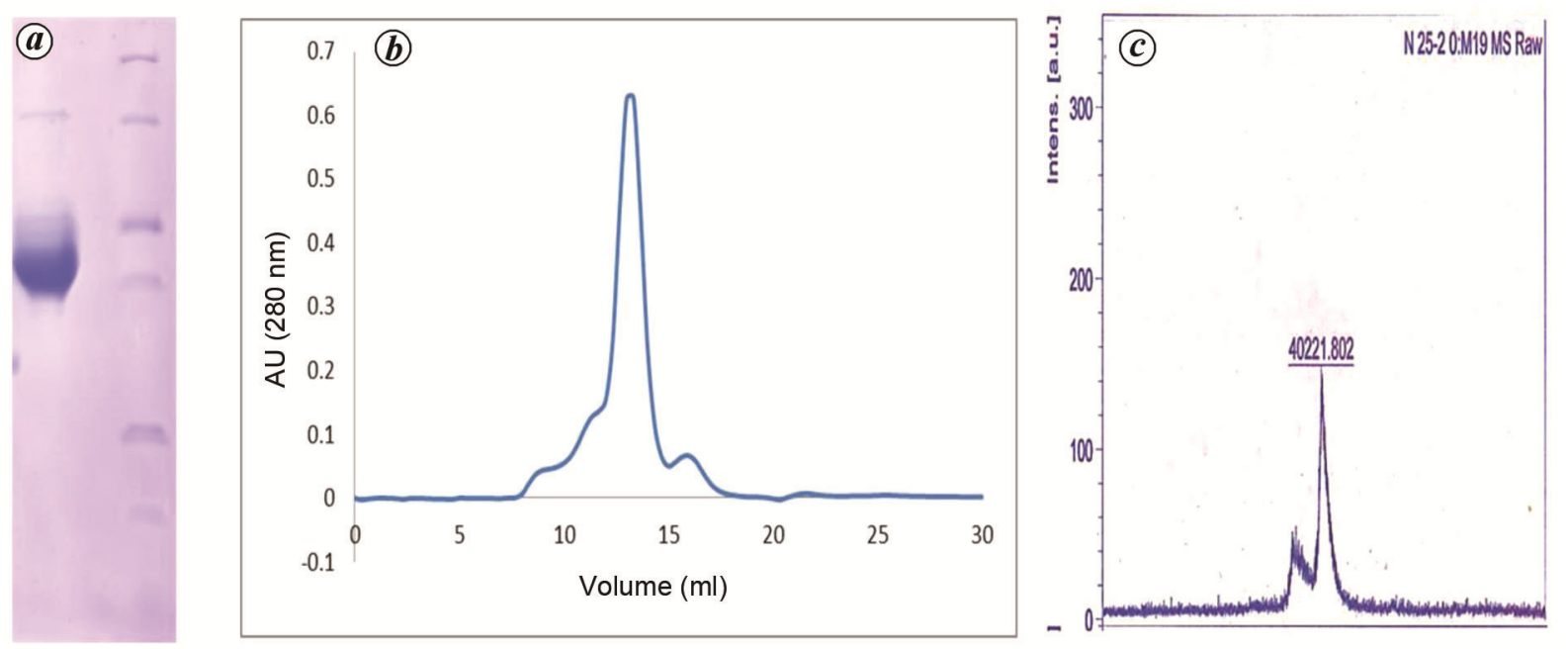

Figure 1. a, SDS-PAGE of PlOXDC. Lane 1 with a band corresponding to $\sim 40 \mathrm{kDa}$ protein represents purified $P l O X D C$, while lane 2 corresponds to molecular weight markers $(116,66,45,35,25$ and $18 \mathrm{kDa})$. $\boldsymbol{b}$, Gel filtration profile showing a predominant peak eluting at $13.5 \mathrm{ml}$ and a much weaker peak eluting at $16 \mathrm{ml}$. The column was standardized using marker proteins from Sigma Aldrich $(200,150,120$ 66 and $29 \mathrm{kDa}$ ). The major peak corresponds to the trimeric form of the enzyme, while the weak peak corresponds to the monomeric species. $c$, MALDI-TOF mass spectrum indicating the protomeric molecular weight as $\sim 40 \mathrm{kDa}$.
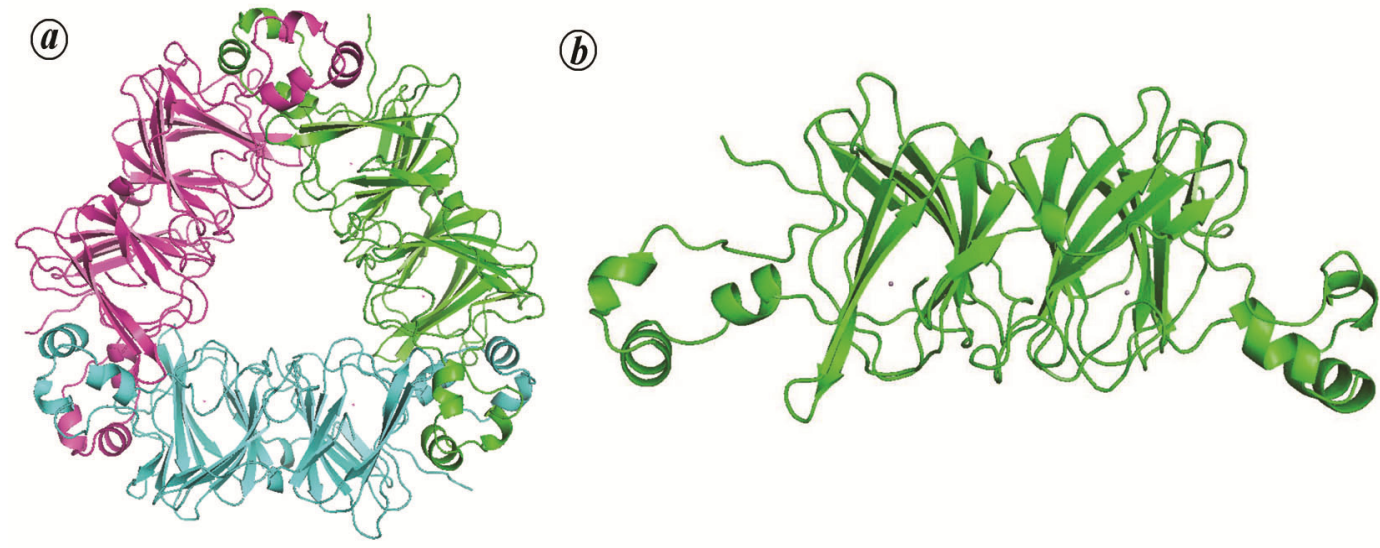

Figure 2. a Trimeric structure of $P l O X D C$. The three chains $A, B$ and $C$ are shown in unique colours. The interlocking of $\mathrm{N}$ - and $\mathrm{C}$-terminal regions of neighbouring protomers of the trimer can be observed. $\boldsymbol{b}$, Ribbon diagram illustrating the bicupin structure of the OXDC protomer. The small dots represent $\mathrm{Mn}^{2+}$ ions. One $\mathrm{Mn}^{2+}$ ion is bound to each of the two cupin domains.

Each protomer of $P l O X D C$ consists of residues 48-388 (Figure $2 b$ ). The protomer consists of two cupin domains (Figure $2 b$ ). Cupin domain I (residues 59-236; domain on the right side in Figure $2 b$ ) consists of two antiparallel $\beta$-sheets containing seven and four $\beta$-strands respectively, and four $\alpha$-helices. Cupin domain II (domain on the left side in Figure $2 b$ ) comprises residues $47-58$ and $237-$ 388 and like domain I, it consists of two antiparallel $\beta$ sheets. However, the characteristic difference from domain I is that the second $\beta$-sheet has only two strands instead of four strands in domain I. The two missing $\beta$ strands in domain II of PlOXDC are replaced by random coil structures in all the three protomers of the trimeric molecule. Domain II also contains four $\alpha$-helices. The two antiparallel $\beta$-sheets in both the domains form barrel-like $\beta$ sandwich structures, which is a common characteristic of cupin domains. Each cupin domain contains a manganese ion bound between two loops and a $\beta$-strand from each sheet. The homologous structure, $B s \mathrm{OXDC}$ is also a bicupin structure. In contrast, the Thermatoga maritima OXDC (Tm OXDC) is a monocupin structure.

\section{Internal gene duplication}

Structural superposition of the two cupin domains of PlOXDC using COOT resulted in a RMSD of $1.2 \AA$ for 175 equivalent $\mathrm{C} \alpha$ atoms (Figure $3 a$ ). Comparison of the amino acid sequences of the structurally equivalent $\mathrm{C} \alpha$ atoms shows an identity of $36 \%$, indicating that the bicupin structure is a result of gene duplication. Similar gene duplication has been observed in several seed storage proteins, phosphomannose isomerase and BsOXDC. 

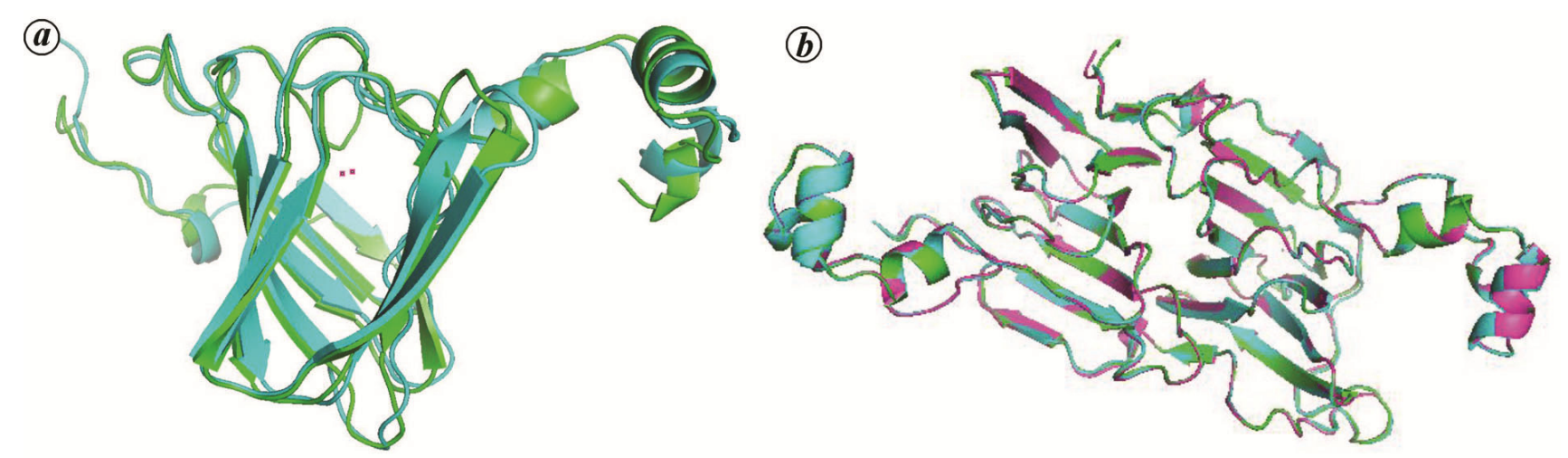

Figure 3. a, Structural superposition of the two monocupin domains of the bicupin PlOXDC protomer. The similarity in their sequence and conservation of their three-dimensional folds suggest that they are products of divergent evolution. $\boldsymbol{b}$, Structural superposition of the three crystallographically independent subunits of PlOXDC illustrating no significant structural differences among the three protomers.

PlOXDC shares sequence identities of $49 \%$ and $22 \%$ with OXDC of $B$. subtilis and $T$. maritima respectively. $B s \mathrm{OXDC}$ is a hexameric protein. TmOXDC has a similar organization, although the two identical monocupin protomers of $T m \mathrm{OXDC}$ are structurally equivalent to a single bicupin protomer of $B s \mathrm{OXDC}$, suggesting that the $B s \mathrm{OXDC}$ protomer also has resulted from gene duplication. Interestingly, gene duplication has not occurred in TmOXDC, which is therefore a monocupin domain.

\section{Oligomeric structure of PlOXDC}

Three crystallographically independent protomers $(A, B$ and $C$ shown in green, blue and pink respectively in Figure $2 a$ ) of PlOXDC are structurally similar, as shown by their superposition (Figure $3 b$ ). The RMSD of corresponding $\mathrm{C} \alpha$ atoms upon structural superposition of pairs of these protomers is in the range $0.25-0.30 \AA$. The three $\alpha$-helices of domain I at the $\mathrm{N}$-terminal end of one protomer interact with three $\alpha$-helices at the C-terminal end of domain II of the neighbouring protomer (Figure $2 a$ ). This pattern continues to form a closed trimeric structure. The two monocupin domains of the three protomers are related by intra-molecular twofold axes that are nearly perpendicular to the threefold axis relating the three protomers of PlOXDC. The three-fold symmetry axis runs along a solvent-accessible channel in the crystal. Each protomer interlocks with two other protomers (Figure $2 a$ ) forming a stable trimer.

$B s \mathrm{OXDC}$ is a hexamer in which two trimers interlock through a helical N-terminal segment (residues 8-47) contributed by each protomer. In PlOXDC, no significant electron density was observed for the $47 \mathrm{~N}$-terminal residues. This truncation and disorder of the equivalent $\mathrm{N}$-terminal region could be the reason for the formation of a trimer rather than a hexamer. A similar kind of trimer is observed in the seed storage proteins, where the sequences lack the equivalent $\mathrm{N}$-terminal segment.

\section{Manganese binding}

The metal ion bound to the protein was identified as manganese using X-ray fluorescence (XRF) data collected at ESRF, Grenoble, France (data not shown). Electron density corresponding to two metal ions per protomer could be observed. Each cupin domain is associated with one metal ion. The metal ion is coordinated by a Glu and three His residues. The four metalbinding residues are conserved among all OXDC homologs. The metal co-coordinating residues in PlOXDC are His98, His100, Glu105 and His144 in cupin domain I (Figure 4a) and His278, His280, Glu285 and His324 in cupin domain II (Figure $4 b$ ). The octahedral coordination of the metal ion is satisfied by the two oxygen atoms of the ligand molecule and/or water molecules. The metalatom coordination distances are in the range 1.9-2.3 $\AA$. In all the structures determined, electron density corresponding to EDO or the ligands used in crystallization was observed close to $\mathrm{Mn}^{2+}$ in both cupin domains.

\section{EDO-bound structure of PlOXDC}

In the PlOXDC structure determined at $1.97 \AA$ resolution, significant electron density that fitted EDO was observed at the active sites of both cupin domains of all the three protomers. The two oxygen atoms $\mathrm{O} 1$ and $\mathrm{O} 2$ of EDO form coordinate bonds with $\mathrm{Mn}^{2+}$ ion in both the domains. Apart from the EDO molecules bound at the active site, six other EDO molecules are bound nonspecifically to surfaces of the two protomers. $\mathrm{A} \mathrm{SO}_{4}^{2-}$ ion, probably derived from the crystallization condition, is located in each of the subunits making hydrogen bonds with Arg250 and surrounding water molecules. The $\mathrm{SO}_{4}^{2-}$ also interacts with Lys254 in chain B. However, these sites are not close to the active site represented by the metal ion. Therefore, non-specific binding of EDO or $\mathrm{SO}_{4}$ binding may not be functionally significant. 

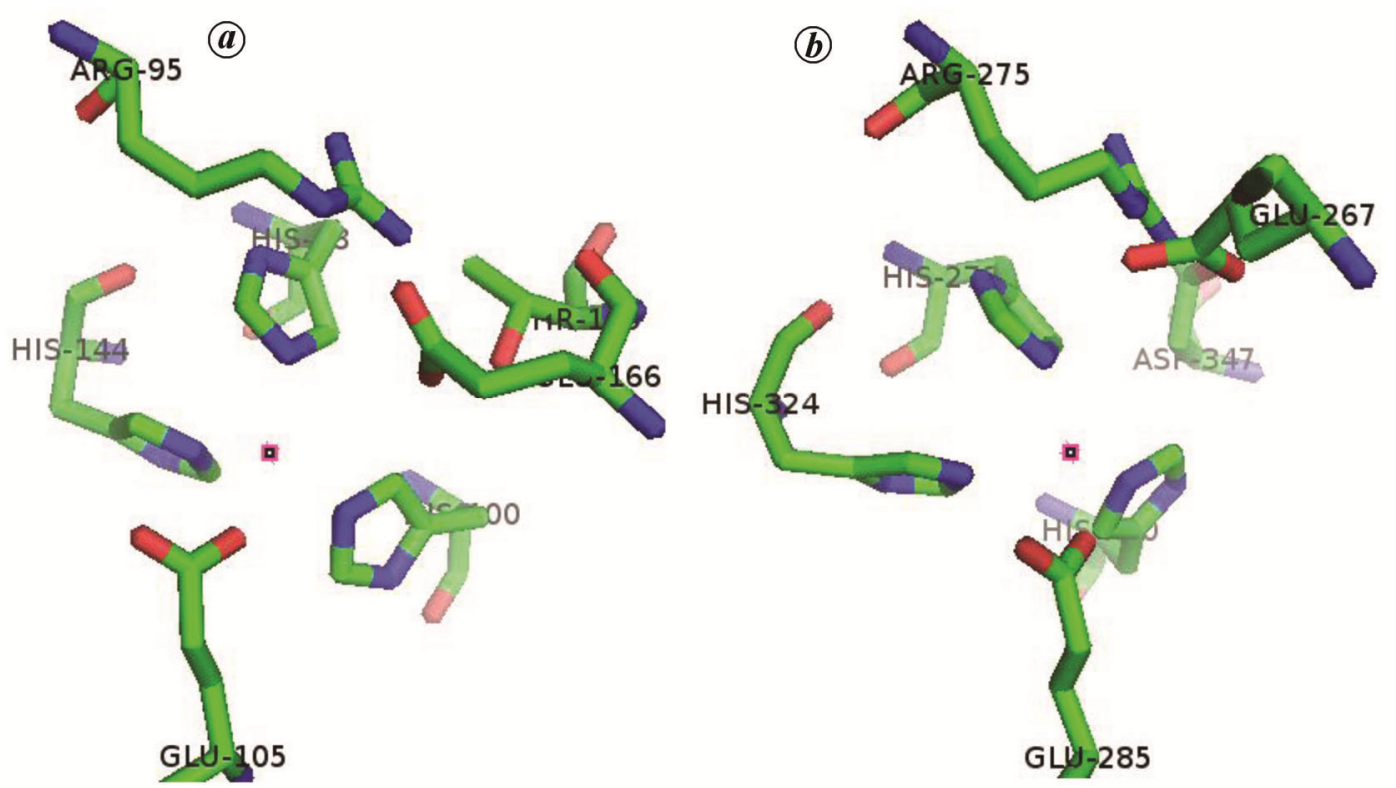

Figure 4. Active site of $P l O X D C$. The residues near the metal ion (small square) are shown in stick representation. The metal-binding residues in (a) cupin domain 1 are H98, H100, H144 and E105 and (b) cupin domain II, are H278, H280, H324 and E267.

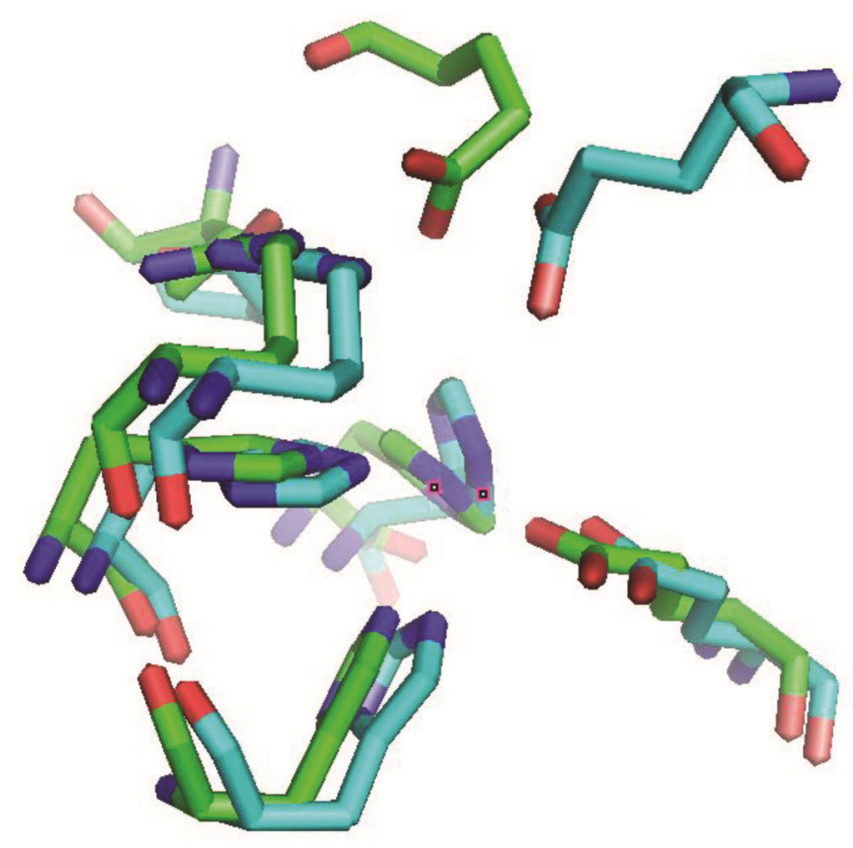

Figure 5. Superposition of cupin domains I and II showing different conformations of E166 and E267. However, the side chain carboxylates are positioned similarly.

In domain I, Arg95 is involved in hydrogen-bonding interaction with Glu166 and Thr169. These interactions appear to control the position of Glu166, which has been proposed as a residue important for catalysis in $B s \mathrm{OXDC}$. The residue in domain II equivalent to Thr169 of domain I is Asp347. This residue interacts with Arg275 (equivalent to Arg95 of domain I), but not with Glu267 (equivalent to Glu166 of domain I). Therefore, the conformations of Glu166 of domain I and the equivalent Glu267 of domain II are not identical. However, the terminal carboxylate groups of Glu166 and Glu267 are in a similar disposition with respect to the active site. Figure 5 shows the superposition of the active sites of cupin domains I and II. It may be observed that the carboxylates of the glutamates are similarly disposed, although their conformations are different. Hence, it will not be surprising if both the cupin domains are catalytically competent.

\section{Discussion}

The X-ray crystal structure of PlOXDC is reported in this article. The homologous $B s \mathrm{OXDC}$ structure was determined by two groups in its formate bound and apo forms respectively. There is an ambiguity as to which of the cupin domains is catalytically active. The first structure of $B s \mathrm{OXDC}$ determined by Anand et al. ${ }^{13}$, had formate bound only in cupin domain I. However, the authors claimed that only cupin domain II was catalytically active since mutation E333A in domain II reduced the activity to $4 \%$ of that of the wild-type enzyme. It was also pointed out that only Glu333 (Glu267 in PlOXDC) is suitably placed to act as a proton donor during oxalate degradation. Also, by mutational analysis, residues Tyr340 and Arg270 in domain II were shown to be essential for catalysis.

On the contrary, Just et al. ${ }^{26}$ argued that Glu162 (Glu166 in PlOXDC) in domain I was a more suitable residue as a proton donor during catalysis based on the structure of the apo form of BsOXDC. They proposed 
that the loop corresponding to residues 161-165 (SENST) acts as a lid, opening and closing the active site during catalysis. In BsOXDC-formate complex determined by Anand et al. ${ }^{13}$, formate was bound to domain I. The presence of formate was assumed by Just et $a l^{26}$ to be the reason why the loop opts for an open conformation in which Glu162 faces away from the active site pocket. They argued that Glu162 could assume a conformation suitable for protonation of the substrate in the closed conformation. Indeed, in $\mathrm{Bs} \mathrm{OXDC}$-apo form ${ }^{26}$, Glu162 faces towards the active site (closed form). In this form, Glu162 could function as a proton donor. Therefore, Just et $a l .{ }^{26}$ proposed that only domain I is catalytically competent. This was also supported by the lack of activity of the mutant E162A. Further, mutation of Arg92 (R92A) was shown to make $B s \mathrm{OXDC}$ inactive. Mutations carried out by Just et $a l .{ }^{26}$ in domain II residues also affected the enzyme activity. E333A showed only 6\% activity (similar to $4 \%$ activity reported by Anand et al. ${ }^{13}$ ) and R270A possessed only $3 \%$ activity when compared to the wildtype enzyme. As both the groups have arrived at the identity of the domain that is catalytically active by careful experimental observations, it is likely that the structural modifications due to mutation in one domain could affect the active site geometry in the other domain accounting for the total loss of activity. Examination of the organization of the N-terminal cupin domain of A-subunit and Cterminal cupin domain of B-subunit showed that the two active sites are indeed tightly connected (Figure 6). The two histidyl moieties that coordinate with the metal atom in both the subunits are separated by a single tryptophan

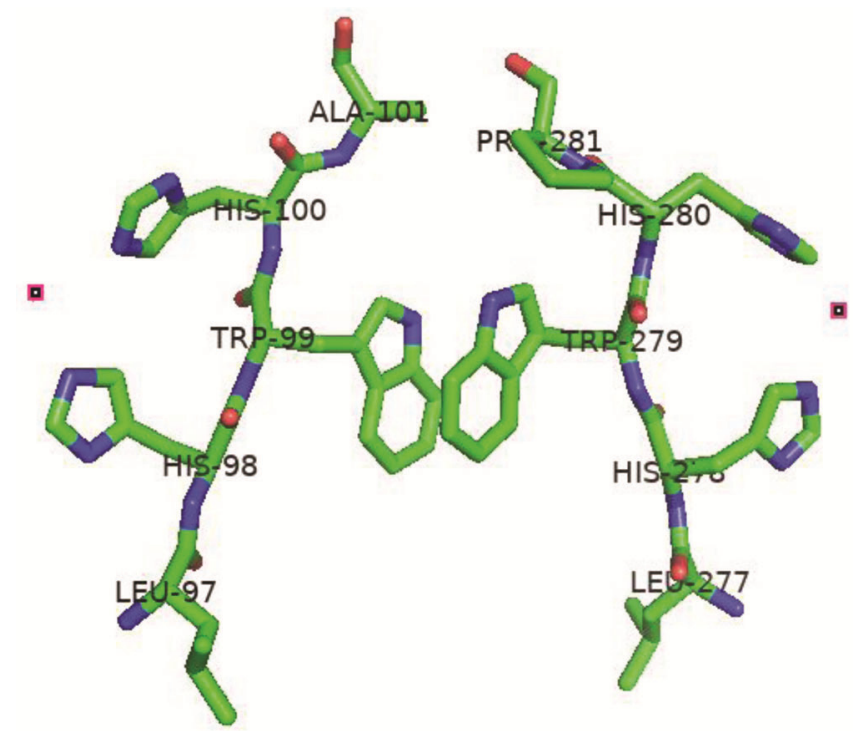

Figure 6. Strong inter-molecular connection between cupin domains of neighbouring subunits of $P l O X D C$ protomers in trimeric structure. The two histidines that coordinate the metal ion are separated by a single tryptophan. The tryptophans from the two neighbouring subunits are tightly connected by $\pi-\pi$ interactions. Therefore, mutation at the active site of one protomer is highly likely to disturb the activity at other active sites as well. residue. These tryptophan residues are held by $\pi-\pi$ interactions. Therefore, any mutation in one of these domains will affect the other domain as well.

In the PlOXDC structure, active site of both the cupin domains contains a glutamate (Glu166 and Glu267) residue which could act as the proton donor for catalysis because of the similar disposition of their terminal carboxylate groups (Figure 6), despite differences in the conformation of the loop hosting the active site glutamate. The arginine residues (Arg95 and Arg275) which are essential for substrate recognition and positioning of Glu for catalysis are also present and involved in hydrogen bonding with the EDO bound at the active site of both cupin domains. In cupin domain I, the interaction between Thr169 and Arg95 is considered to be important for positioning Glu166 (ref. 26). This specific Thr169 is present in the segment 165-169 (SEFGT) and adapts a closed conformation in PlOXDC. This loop corresponds to the SENST loop of $B s \mathrm{OXDC}$. A corresponding threonine residue is absent in domain II of PlOXDC, while an alternative interaction between $\operatorname{Arg} 275$ and Asp347 positions Glu267 (Glu333 in BsOXDC) for catalysis, although the loop closure of domain $\mathrm{I}$ is not observed in domain II. The comparisons presented here suggest that the residues required for oxalate decarboxylation are present in both the domains of PlOXDC, and both the domains may be catalytically competent.

Interestingly, Weerth et al. ${ }^{18}$ have reported the structure of a protein of unknown function from $P$. luminescens subsp. laumondii TTO1 at $1.35 \AA$. The polypeptide corresponding to this dimeric protein has a monocupin fold. However, the active site cavity of the protein has been reported to be wider and could accommodate a substrate larger than oxalate. However, the activity of this protein with either oxalate or its homologs has not been examined. A protomer of this structure could be superposed well with a single domain of PlOXDC protomer. The other protomer of the dimer then gets superposed on the second cupin domain of PlOXDC. This again points to gene duplication as the underlying mechanism in the evolution of bicupin OXDC proteins.

The structure of trimeric PlOXDC described here could serve as a novel insecticidal protein. We speculate that the $\mathrm{Mn}^{2+}$ dependent OXDC secreted by P. luminescens could cause tissue and cell death by forming excess quantities of formate and $\mathrm{CO}_{2}$ in the hemolymph (insect blood in body cavity) that are toxic to the insects. The products of the OXDC-catalysed reaction could also affect the physiological functions, immunity and tissue necrosis of the host ${ }^{27}$. Creation of anaerobic condition by the production of $\mathrm{CO}_{2}$ could ward-off aerobic microbes and prevent putrefaction. Thus, OXDC could play an important role in insect physiology and nematode-bacterium-insect host tritrophic interactions, and may serve as a biocontrol agent for insect pest management. 


\section{Conclusions}

X-ray crystal structure of OXDC from Photorhabdusluminescens was determined the at $1.97 \AA$ resolution. Each protomer of OXDC consists of two cupin domains. Each cupin domain consists of two antiparallel $\beta$ sheets organized as a sandwich with a $\mathrm{Mn}^{2+}$ ion bound at the active site. In contrast to the hexameric $B s \mathrm{OXDC}, P l \mathrm{OXDC}$ exists as a mixture of monomeric and trimeric forms in solution but assumes a trimeric form in the crystal structure probably due to the cleavage of a $3 \mathrm{kDa}$ peptide from the N-terminus of intact PlOXDC. Despite this cleavage, PlOXDC retains catalytic activity. The active sites of neighbouring subunits in the trimeric $P l O X D C$ are tightly linked by the interaction between the $\mathrm{C}$-terminal cupin domain of a protomer and the $\mathrm{N}$-terminal cupin domain of a neighbouring protomer. This association accounts for the results of earlier studies on the enzyme where activity of both the cupin domains was lost upon mutation of an active site residue in only one of the protomers. Comparison of BsOXDC and PlOXDC structures suggests that both cupin domains of the protomer are likely to be catalytically competent.

1. Fischer-Le Saux, M., Viallard, V., Brunel, B., Normand, P. and Boemare, N. E., Polyphasic classification of the genus Photorhab$d u$ and proposal of new taxa: P. luminescens subsp. luminescens subsp. nov., P. luminescens subsp. akhurstii subsp. nov., P. luminescens subsp. laumondii subsp. nov., P. temperata sp. nov., $P$. temperata subsp. temperata subsp. nov. and $P$. asymbiotica $\mathrm{sp}$. nov. Int. J. Syst. Evol. Microbiol., 1999, 49, 1645-1656.

2. $\mathrm{Hu}, \mathrm{K}$. and Webster, J. M., Antibiotic production in relation to bacterial growth and nematode development in PhotorhabdusHeterorhabditis infected Galleria mellonella larvae. FEMS Microbiol. Lett., 2000, 189, 219-223.

3. Duchaud, E. et al., The genome sequence of the entomopathogenic bacterium Photorhabdus luminescens. Nature Biotechnol., 2003, 21, 1307-1313.

4. Shimazono, H., Oxalic acid decarboxylase, a new enzyme from the mycelium of wood destroying fungi. J. Biochem., 1955, 42, 321-340.

5. Dutton, M. V., Kathiara, M., Gallagher, I. M. and Evans, C. S., Purification and characterization of oxalate decarboxylase from Coriolus versicolor. FEMS Microbiol. Lett., 1994, 116, 321-325.

6. Emiliani, E. and Riera, B., Enzymatic oxalate decarboxylation in Aspergillus niger: II. Hydrogen peroxide formation and other characteristics of the oxalate decarboxylase. Biochim. Biophys. ActaEnzymol., 1968, 167, 414-421.

7. Kathiara, M., Wood, D. A. and Evans, C. S., Detection and partial characterization of oxalate decarboxylase from Agaricus bisporus. Mycol. Res., 2000, 104, 345-350.

8. Lillehoj, E. B. and Smith, F. G., An oxalic acid decarboxylase of Myrothecium verrucaria. Arch. Biochem. Biophys., 1965, 109, 216-220.

9. Lung, H.-Y., Cornelius, J. G. and Peck, A. B., Cloning and expression of the oxalyl-CoA decarboxylase gene from the bacterium, Oxalobacter formigenes: prospects for gene therapy to control Ca-oxalate kidney stone formation. Am. J. Kidney Dis., 1991, 17, 381-385.

10. Magro, P., Marciano, P. and Di Lenna, P., Enzymatic oxalate decarboxylation in isolates of Sclerotinia sclerotiorum. FEMS Microbiol. Lett., 1988, 49, 49-52.
11. Mehta, A. and Datta, A., Oxalate decarboxylase from Collybia velutipes. Purification, characterization, and cDNA cloning. J. Biol. Chem., 1991, 266, 23548-23553.

12. Shimazono, H. and Hayaishi, O., Enzymatic decarboxylation of oxalic acid. J. Biol. Chem., 1957, 227, 151-159.

13. Anand, R., Dorrestein, P. C., Kinsland, C., Begley, T. P. and Ealick, S. E., Structure of oxalate decarboxylase from Bacillus subtilis at $1.75 \AA$ A resolution. Biochemistry, 2002, 41, 7659-7669.

14. Schwarzenbacher, R. et al., Crystal structure of a putative oxalate decarboxylase (TM1287) from Thermotoga maritima at $1.95 \AA$ resolution. Proteins, 2004, 56, 392-395.

15. Dunwell, J. M., Khuri, S. and Gane, P. J., Microbial relatives of the seed storage proteins of higher plants: conservation of structure and diversification of function during evolution of the cupin superfamily. Microbiol. Mol. Biol. Rev., 2000, 64, 153-179.

16. Dunwell, J. M., Purvis, A. and Khuri, S., Cupins: the most functionally diverse protein superfamily? Phytochemistry, 2004, 65, $7-17$.

17. Woo, E.-J., Dunwell, J. M., Goodenough, P. W., Marvier, A. C. and Pickersgill, R. W., Germin is a manganese containing homohexamer with oxalate oxidase and superoxide dismutase activities. Nature Struct. Biol., 2000, 7, 1036-1040.

18. Weerth, R. S. et al., Structure of a cupin protein Plu4264 from Photorhabdus luminescens subsp. laumondii TTO1 at $1.35 \AA$ resolution. Proteins, 2015, 83, 383-388.

19. Dean, D. H., Biochemical genetics of the bacterial insect-control agent Bacillus thuringiensis: basic principles and prospects for genetic engineering. Biotechnol. Genet. Eng. Rev., 1984, 2, 341363.

20. Bergmeyer, H. and Grass1, M., Methods of Enzymatic Analysis, Verlag Chemie, Weinheim, Germany, 1983, 3rd edn, pp. 267-268.

21. Potterton, E., Briggs, P., Turkenburg, M. and Dodson, E., A graphical user interface to the CCP4 program suite. Acta Crystal$\log r$. D, 2003, 59, 1131-1137.

22. McCoy, A. J., Grosse-Kunstleve, R. W., Adams, P. D., Winn, M. D., Storoni, L. C. and Read, R. J., Phaser crystallographic software. J. Appl. Crystallogr., 2007, 40, 658-674.

23. Otwinowski, Z. and Minor, W., Processing of X-ray diffraction data. Methods Enzymol., 1997, 276, 307-326.

24. Emsley, P., Lohkamp, B., Scott, W. G. and Cowtan, K., Features and development of Coot. Acta Crystallogr. D, 2010, 66, 486-501.

25. Murshudov, G. N. et al., REFMAC5 for the refinement of macromolecular crystal structures. Acta Crystallogr. D, 2011, 67, 355367.

26. Just, V. J., Stevenson, C. E., Bowater, L., Tanner, A., Lawson, D. M. and Bornemann, S., A closed conformation of Bacillus subtilis oxalate decarboxylase OxdC provides evidence for the true identity of the active site. J. Biol. Chem., 2004, 279, 19867-19874.

27. Held, K. G., LaRock, C. N., D'argenio, D. A., Berg, C. A. and Collins, C. M., A metalloprotease secreted by the insect pathogen Photorhabdus luminescens induces melanization. Appl. Environ. Microbiol., 2007, 73, 7622-7628.

ACKNOWLEDGEMENTS. S.C. thanks University Grants Commission, Government of India (GoI) for the D. S. Kothari Postdoctoral Fellowship. H.S.S. and M.R.N. thank Department of Science and Technology (DST), Department of Biotechnology (DBT), GoI for financial support and DST for the award of Bose Fellowships. H.S.S., M.N. and R.T.S. thank DBT and ICAR-NBAIR for supporting related work. H.S.S. also thanks the National Academy of Sciences, India for the Senior Scientist position.

Received 10 May 2020; revised accepted 4 August 2020

doi: $10.18520 / \mathrm{cs} / \mathrm{v} 119 / \mathrm{i} 8 / 1349-1356$ 